\title{
Statistics of Transmission Eigenvalues in Two-Dimensional Quantum Cavities: Ballistic versus Stochastic Scattering
}

\author{
Stefan Rotter, ${ }^{1,2}$ Florian Aigner, ${ }^{1}$ and Joachim Burgdörfer ${ }^{1}$ \\ ${ }^{1}$ Institute for Theoretical Physics, Vienna University of Technology, A-1040 Vienna, Austria, EU \\ ${ }^{2}$ Department of Applied Physics, Yale University, New Haven, CT 06520, USA
}

(Dated: September 14, 2018)

\begin{abstract}
We investigate the statistical distribution of transmission eigenvalues in phase-coherent transport through quantum dots. In two-dimensional ab-initio simulations for both clean and disordered twodimensional cavities, we find markedly different quantum-to-classical crossover scenarios for these two cases. In particular, we observe the emergence of "noiseless scattering states" in clean cavities, irrespective of sharp-edged entrance and exit lead mouths. We find the onset of these "classical" states to be largely independent of the cavity's classical chaoticity, but very sensitive with respect to bulk disorder. Our results suggest that for weakly disordered cavities the transmission eigenvalue distribution is determined both by scattering at the disorder potential and the cavity walls. To properly account for this intermediate parameter regime we introduce a hybrid crossover scheme which combines previous models that are valid in the ballisic and the stochastic limit, respectively.

PACS numbers: 73.23.-b, 05.45.Mt, 73.63.Kv, 72.70.+m
\end{abstract}

Shot noise, i.e. the fluctuations of the current due to the statistical nature of charge transport, has recently become an intensively studied subject-matter in the field of mesoscopic physics. Being first investigated on a macroscale now almost a century ago $\frac{1}{\underline{n}}$ the interest in this phenomenon has recently witnessed a revival (see Ref. 2 for an introduction to this topic and Ref. 3 for an extensive review). On the experimental side, modern semiconductor fabrication techniques have allowed for high-precision experiments of quantum shot noise $\frac{4,5,6,7,8.9}{\text { On }}$ the theoretical side it was demonstrated that these measurements allow to extract detailed information on microscopic transport mechanisms which are difficult to access

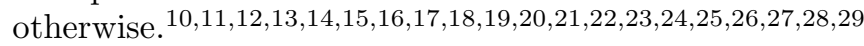
$30,31,32$

Since shot noise on the mesoscopic scale is due to the quantum (probabilistic) nature of transport, a suppression of shot noise has been predicted ${ }^{16}$ as transport becomes more classical (or deterministic), i.e. when the ratio of the Fermi wavelength $\lambda_{F}$ to the linear cavity size $L$ vanishes, $\lambda_{F} / L \rightarrow 0$. Whereas this predic-

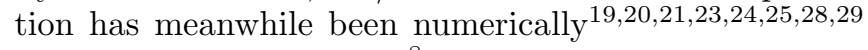
as well as experimentally ${ }^{-8}$ confirmed, it is still a subject of debate how to identify signatures of the different sources of noise in this quantum-to-classical crossover. On the theoretical side, different analytical predictions describe the quantum-to-classical crossover for cavities with ballistic scattering (off smooth potential or boundary profiles) $16,22,23,24$ or with disorder scattering (off short-range impurities or rough boundaries) $\stackrel{17,26}{=}$ In ballistic dots the crossover to the noiseless classical regime is anticipated to be mediated by "noiseless scattering states" $\underline{22}$ The separation of phase space in noiseless classical (i.e. deterministic) and noisy quantum channels is in sharp contrast to the case of cavities with bulk disorder where all transporting channels are expected to contribute to shot noise $\stackrel{15,24,25,26,28,33}{ }$ Testing the valid- ity of these theories has turned out to be a major challenge: Measurements suffer from limited accuracy and seem to be able to explore only the onset of the quantumto-classical crossover where different models are difficult to distinguish from each other $\stackrel{\underline{8}}{*}$ Also numerical simulations for two-dimensional (2D) transport $19,20,21,25,30$ suffer from slow convergence for $\lambda_{F} / L \rightarrow 0$, which reason has prevented a detailed test of differing predictions in that limit. To circumvent this problem an open dynamical kicked rotator model has recently been used to mimic chaotic as well as stochastic scattering in a 1D system. 23,24,28,29 While being computationally more easily tractable, especially in the semiclassical regime of small $\lambda_{F}$, these stroboscopic models do, however, not fully incorporate features of $2 \mathrm{D}$ transport which contribute significantly to the shot noise - as, e.g., whispering gallery modes 20 and an accurate description of diffraction at the dot openings or at a bulk disorder potential. $\stackrel{25,30}{.30}$

The aim of the present communication is to provide such a $2 \mathrm{D}$ transport simulation in the quantumto-classical crossover regime. Our calculations are performed within the framework of the modular recursive Green's function method (MRGM) $\stackrel{34}{=}$ In our singleparticle model, effects of finite temperature and electronelectron interaction are neglected. Finite temperatures would lead to a cross-over from shot noise to thermal noise and inelastic electron-electron interactions would increase the noise ${ }^{14.17}$ The effect of both mechanisms can however be controlled in the experiment by reducing temperature and system size down to a regime where inelastic scattering sources can be neglected. ${ }^{4,6.7}$ We study cavities with $N$ open channels in each of the two attached leads of equal width (injection from the left) and characterize the transport problem by the transmission $(t)$ and reflection $(r)$ matrices of dimension $N \times N$. Following the Landauer-Büttiker theory, the transmission eigenvalues $T_{n}$ of the matrix $t^{\dagger} t$ determine the average 
current, $\langle I\rangle=\Delta \mu \sum_{n} T_{n}$ and the shot noise power, ${ }^{12}$ $S \equiv\left\langle\left\langle I^{2}\right\rangle\right\rangle=\Delta \mu \sum_{n} T_{n}\left(1-T_{n}\right.$ ) (assuming $e=h=1$, and a chemical potential difference $\Delta \mu$ between the two leads). Since also all higher cumulants of the current, $\left\langle\left\langle I^{m}\right\rangle\right\rangle$, are determined by the eigenvalues $T_{n}$, knowledge of the distribution function of the eigenvalues, $P(T)$, allows to obtain the full counting statistics of the transport problem ${ }^{35}$ The distribution $P(T)$ will be at the center of our attention in the present article as different mechanisms of transport leave conspicuous signatures on its functional form. It was first pointed out for the case of a diffusive wire, that this system's eigenvalue distribution function features a bimodal distribution with maxima near values of very high $(T \approx 1)$ and very low $(T \approx 0)$ transmission, respectively $\stackrel{10,11}{=}$ The effects of this feature on the suppression of noise have been predicted 13 and were successfully measured in the experiment, $\stackrel{4,5,6}{\stackrel{6}{a}}$ as well as simulated numerically $\underset{21}{\underline{w}}$ For classically chaotic rather than diffusive systems with $N \gg 1$ and time reversal symmetry, random matrix theory (RMT) predicts $P(T)$ to follow also a bimodal universal form,, 36

$$
P_{R M T}(T)=\pi^{-1}[T(1-T)]^{-1 / 2}, \quad T \in[0,1] .
$$

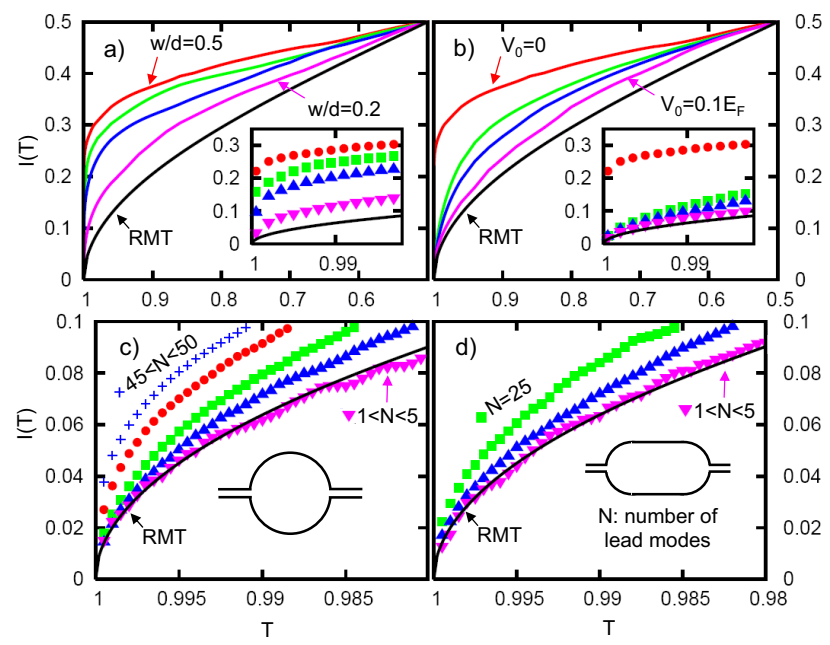

FIG. 1: (Color online) Integrated distribution function of transmission eigenvalues, $I(T)$. Top row: Rectangular billard with tunable shutters (see inset Fig. 2). (a) Crossover from large to small shutter openings (at zero disorder, $V_{0}=$ 0 ): $\mathrm{I}(\mathrm{T})$ for $w / d=0.5,0.4,0.3$, and 0.2 (top to bottom). (b) Crossover from clean to disordered samples (at halfopening, $w / d=0.5)$ : $\mathrm{I}(\mathrm{T})$ for different disorder potentials $V_{0}=0,0.03 E_{F}, 0.05 E_{F}$, and $0.1 E_{F}$ (top to bottom). The pronounced difference between (a) and (b) near $T=1$ is highlighted in the insets. Bottom row: $I(T)$ for the circular (c) and the stadium-shaped geometry (d) for different values of $k_{F}$. In (c) we average over the intervals $45<N<50$, $30<N<35,20<N<25,10<N<15$ and $1<N<5$ (top to bottom), in (d) $N=25,10<N<15,1<N<5$ (top to bottom), where $N$ is the number of open lead modes. The RMT limit is indicated by black lines.
For ballistic cavities the quantum-to-classical crossover of this eigenvalue distribution is predicted to proceed via "noiseless states", 22,24

$$
P^{\alpha}(T)=\alpha P_{R M T}(T)+(1-\alpha)[\delta(T)+\delta(1-T)] / 2 .
$$

Noiseless (or deterministic) transport channels with eigenvalues 0 or 1 and weight $(1-\alpha)$ [represented by the last two terms in Eq. (2)] are expected to appear as soon as classical transmission bands ${ }^{38}$ in phase space can be resolved by the quantum scattering process $\stackrel{22}{2}$ For a chaotic system the continuous crossover parameter $\alpha \in(0,1)$ was predicted $^{16}$ to scale as $\alpha=\exp \left(-\tau_{E} / \tau_{D}\right)$ with $\tau_{D}$ being the dwell time and $\tau_{E}$ the Ehrenfest time in the cavity. The latter estimates the time that it takes for a well-localized quantum wave packet to spread to the size $d$ of the cavity $(d \approx \sqrt{A}$ with $A$ the area of the dot) due to diverging classical trajectories. With the help of the Lyapunov exponent $\Lambda$, which measures the rate of this divergence, the Ehrenfest time is typically estimated as: ${ }^{37} \tau_{E} \approx \Lambda^{-1} \ln \left(d / \lambda_{F}\right)$. Note, however, that this estimate requires corrections for regular or weakly chaotic systems $\frac{25}{2}$

In the presence of a uniform disorder with a correlation length smaller than the electron wavelength $\lambda_{F}$ ("shortrange bulk disorder") the formation of noiseless states is suppressed by stochastic scattering. Also the trajectorybased concept of the Ehrenfest-time as a crossover parameter breaks down here, leading to a different crossover form, $\stackrel{26}{\underline{1}}$

$$
P^{\beta}(T)=P_{R M T}(T) \ln \beta \int_{-1}^{1} d u \frac{(1-u)^{2}|u|^{-(1+2 \ln \beta)}}{4 T u-(1+u)^{2}} .
$$

The crossover parameter $\beta=\exp \left(-\tau_{Q} / \tau_{D}\right) \in(0,1)$ features the characteristic scattering time $\tau_{Q}$ which measures the time within which an initially well-localized wave packet is stochastically scattered into random direction. Note that the stochastic crossover, Eq. (3), interpolates between the same limiting cases $P_{R M T}$ (for $\beta \rightarrow 1$ ) and $P_{c l}=[\delta(T)+\delta(1-T)] / 2$ (for $\beta \rightarrow 0$, i.e. vanishing disorder) as the ballistic crossover in Eq. (2).

We now search for signatures of these two crossover scenarios in the numerical results for $P(T)$. To this end we calculate transport through a rectangular cavity (see inset Fig. 2) with area $d \times 2 d$ and two tunable openings ("shutters") of width $w$ (inspired by recent shot noise experiments ${ }^{8}$ ). The cavity interior contains a static bulk disorder potential $V$ with a mean value $\langle V\rangle=0$ and a correlation function $\langle V(x) V(x+a)\rangle=$ $\left\langle V^{2}\right\rangle \exp \left(-a / l_{C}\right)$. The correlation length $l_{C}$ is smaller than the Fermi wavelength, $l_{C} / \lambda_{F} \approx 0.12$, and the potential strength $V_{0}=\sqrt{\left\langle V^{2}\right\rangle}$ corresponds to moderate disorder, $V_{0} \in[0,0.1] \times E_{F}$ (for details on the disorder potential see Ref. 25). In the limit of vanishing disorder strength $\left(V_{0} \rightarrow 0\right)$ the motion inside the rectangular cavity becomes completely regular.

We calculate 400 equidistant points in the interval $k_{F} \in[40.1,40.85] \times \pi / d$. In order to better resolve the 
behavior of $P(T)$ near $T=1$ we plot the integrated eigenvalue distribution $24,26 I(T)=\int_{T}^{1} P(\tau) d \tau$. For cavity parameters favorable to the appearance of noiseless scattering channels, i.e. vanishing disorder $\left(V_{0}=0\right)$, large openings $(w=d / 2)$, and large $k_{F}$, we find that $I(T)$ features a very pronounced offset at $T \approx 1$ (see Fig. 1 a), corresponding to a statistically significant portion of effectively noiseless eigenvalues $T>0.999$. To verify whether these "classical" transmission eigenvalues are indeed due to direct scattering processes, we control their weight by gradually decreasing the cavity openings $w$ (Fig. 1a). Reducing $w$ decreases the offset and gradually shifts the distribution $P(T)$ towards its RMT-limt [Eq. (1)] for $w \rightarrow 0$. This behavior is all the more interesting as our sharp cavity openings do give rise to diffractive scattering 25,38 which might suppress the formation of noiseless states. Our observation suggests, however, that noiseless transmission can still occur when scattering states effectively bypass any diffractive corners 26.28 To further test this hypothesis we now gradually turn on the bulk disorder strength up to values of $V_{0}=0.1 \times E_{F}$. Bulk disorder cannot be bypassed by any transmitting state and should therefore destroy the noiseless channels and, consequently, the offset in $I(T)$. We find that already a small disorder potential $\left(V_{0}=0.03 \times E_{F}\right)$ suppresses the offset in $I(T)$ entirely (Fig. 1b). With higher values of $V_{0}$ we reach the RMT-limit for $I(T)$. The striking dif-

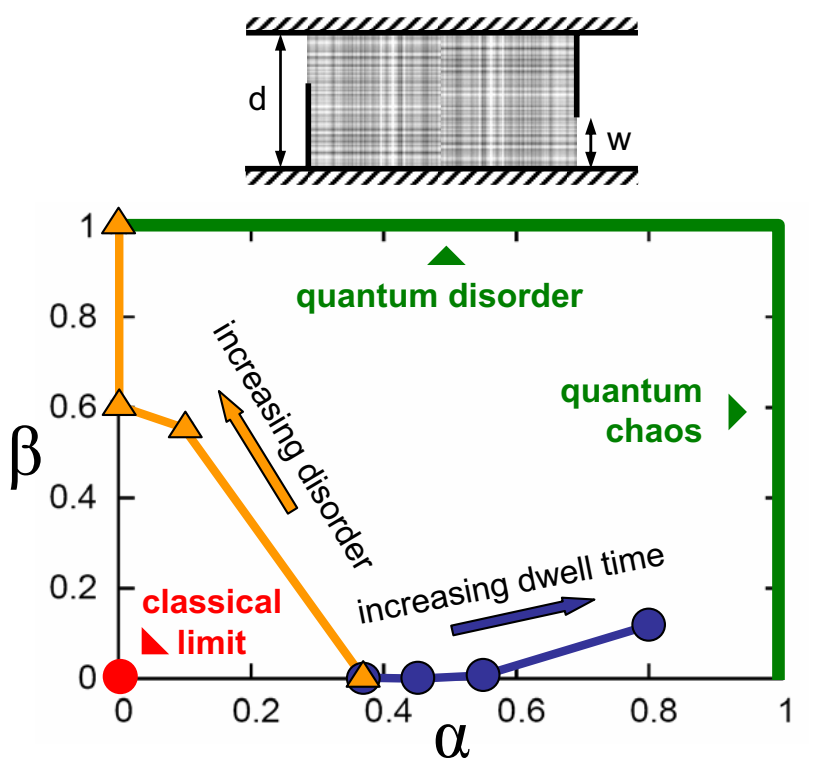

FIG. 2: (Color online) Evolution of the crossover trajectories in the parameter space $(\alpha, \beta)$, obtained by fitting Eq. (4) to the data displayed in Fig. 1a,b. Starting parameters of both trajectories: $w / d=0.5, V_{0}=0$. Trajectory 1 for fixed $w / d=0.5$ (orange triangles): $V_{0}=0.03 E_{F}, 0.05 E_{F}$ and $0.1 E_{F}$. Trajectory 2 for fixed $V_{0}=0$ (blue circles): $w / d=0.4$, 0.3 and 0.2 . Inset: Rectangular cavity with tunable shutter openings and disorder strength. ference between the ballistic (Fig. 1 $\mathrm{a}$ ) and the stochastic crossover (Fig. 1 $\mathrm{b}$ ) is best visualized by zooming into the distribution $I(T)$ at values close to $T=1$ where the gradual vs. "sudden" suppression of the offset becomes most apparent (see insets in Figs. 1 $1 \mathrm{a}, \mathrm{b}$ ). The observation that $I(T)$ depends on the specific character of the diffractive scattering ("bulk vs. surface disorder") is in line with recent investigations $\frac{15,24,25,26,33}{2}$ To the best of our knowledge, the present results explicitly demonstrate for the first time in a genuine $2 \mathrm{D}$ system, how these different noise sources influence the emergence of noiseless scattering states.

To analyze our findings quantitatively, we compare our numerical results for the eigenvalue distribution $P(T)$ with the analytical predictions of Eqs. (223). Note, however, that in our cavities there will always be contributions from ballistic and stochastic scattering sources, rather than from either source alone. Stochastic scattering events do occur for any variation of the potential on a scale smaller than $\lambda_{F}$, such as sharp cavity openings or short-range bulk disorder. Ballistic broadening of wave packets is induced by scattering at the cavity walls which, except for the openings, are always chosen to be suffienctly "smooth". Particularly in the regime of weak disorder both mechanisms will leave their signatures on the transmission eigenvalue distribution. To properly account for these signatures we propose to merge the crossover models Eqs. (223) in the following way: We start from the crossover model for a ballistic system, $P^{\alpha}(T)[$ Eq. (2)], which correctly describes the appearance of noiseless channels in the absence of bulk disorder (Fig. 1 $\mathrm{a}$ ). Introducing disorder is expected to affect (a) the noisy as well as (b) the noiseless part of $P^{\alpha}(T)$ and will furthermore induce (c) flux exchange processes between these two components. The effect of the disorder on (a) and (b) is suggested by Eq. (3): Whereas the indeterministic, intrinsically noisy channels $P_{R M T}(T)$ in (a) should remain unchanged, the deterministic distribution $P_{c l}(T)$ in (b) is expected to evolve as described by $P^{\beta}(T)$ in Eq. (3). The flux exchange between the two phase-space components (c) is stochastic and should mutually balance. This suggests the following crossover model for cavities with both ballistic and stochastic scattering sources,

$$
P^{\alpha, \beta}(T)=\alpha P_{R M T}(T)+(1-\alpha) P^{\beta}(T) .
$$

This "hybrid" crossover model should serve as a good starting point for analyzing the case of weak disorder scattering, $\stackrel{40}{,}$ allowing us to quantify the crossovers (Fig. 1, b) in the 2D parameter space of $\alpha, \beta \in(0,1)$ (see Fig. 21). Note that the classical (i.e. deterministic) limit in this $2 \mathrm{D}$-space corresponds to the point $(\alpha=\beta=0)$, whereas the quantum or RMT limit is represented by the lines of the parameter space at $\alpha=1$ (ballistic "quantum chaos") and at $\beta=1$ (stochastic "quantum disorder"). For the shot noise Fano factor $F$ our hybrid model translates to the crossover $F \approx(1 / 4) \times(1-\alpha \ln \beta) /(1-\ln \beta)$, thereby reproducing $F \approx \alpha / 4$ in the absence of stochas- 
tic scattering 16 ( $\beta \rightarrow 0)$ and $F \approx(1 / 4) /(1-\ln \beta)$ in the absence of ballistic scattering $26(\alpha \rightarrow 0)$. Fitting our numerical results for $P(T)$ (Fig. 1a,b) by Eq. (4) allows to describe the crossover in terms of different trajectories in the $(\alpha, \beta)$ parameter space. In the absence of bulk disorder, the trajectory for decreasing shutter openings (i.e. increasing dwell time) features small $\beta$ as it approaches the RMT limit. On the other hand, increasing the disorder potential $V_{0}$ results in the approach of the RMT limit through a rapid increase in $\beta$ while $\alpha$ tends to zero (indicating the mergence of the two separate phase space components (a),(b) for increasing $V_{0}$ ). Note that with Eq. (4) we can directly quantify the signatures that either ballistic or stochastic scattering in $2 \mathrm{D}$ cavities leave on $P(T)$.

At this point the question suggests itself, whether the above differences in the crossover behavior leave clear signatures in any of the current cumulants $\left\langle\left\langle I^{m}\right\rangle\right\rangle$ that might be accessible experimentally. For "symmetric" cavities with an equal number of incoming and outgoing channels we have found already previously 25 that such differences are hard to pin down in the shot noise (i.e. in the second cumulant, $m=2$ ). A straigtforward evaluation of $\left\langle\left\langle I^{m}\right\rangle\right\rangle$ for all $m$ [using our numerical data from Fig. 19,b or, alternatively, Eqs. (23)], reveals that the above differences in the crossover do not lead to characteristic signatures in any of the individual current cumulants. Rather than appearing in individual cumulants explicitly, the characteristic differences in the values of $P(T)$ near $T \approx 0,1$ seem to be distributed over all even cumulants of the current (the odd cumulants are strongly suppressed due to the symmetry of $P(T)$ with respect to $\langle T\rangle \approx 0.5)$. A possible strategy to circumvent this limitation would be to resort to cavities with different degrees of opening to the left and right reservoir $\frac{18,26,27}{}$ In such "asymmetric" cavities the internal cavity dynamics is expected to leave clear signatures already on the third cumulant, a quantity which recently could be accessed experimentally in tunnel junctions $\frac{9}{9}$

We now probe for the influence of the underlying chaotic classical dynamics on the transmission eigenvalue statistics at vanishing bulk disorder (following previous investigations on shot noise $19,20,25,30)$. To this end we contrast the transport properties of a circular and a stadium billiard (see insets Figs. 11,d). Due to the classical scaling invariance of ballistic billiards with constant potential in the interior, we can probe the quantum-toclassical crossover, $\hbar_{\mathrm{eff}} \rightarrow 0$, by the limit $k_{F} \rightarrow \infty$. Although numerically very demanding, we study the regime 41 of comparatively long dwell time $\tau_{D}$ to assure a sufficiently "universal" behavior. For the circular billiard we can reach 50 open lead modes, whereas for technical reasons only half as many modes can be accessed for the stadium. 34 Both geometries feature a fourfold symmetry, for which case $P_{R M T}$ [Eq. (1)] applies also for low mode numbers ${ }^{32} N$. Deviations from $P_{R M T}$ can therefore be interpreted as contributions of noiseless channels.

Remarkably, for the low-energy interval $k_{F} \in[1,5] \pi / d$, we find excellent agreement between the numerical distribution $I(T)$ and its RMT-prediction, for both the stadium and the circle billiard (see Fig. 11,d). Differences between regular and chaotic dynamics do not leave any imprint on $I(T)$. At higher electron energies (or smaller wavelengths) the onset of noiseless scattering is similarly reflected in $I(T)$ for both geometries (Fig. 1k,d), irrespective of the classical chaoticity or the lack thereof. This finding points to the conclusion that the appearance of the first noiseless states is uniquely determined by the requirement that quantum mechanics can resolve the largest classical transmission band in phase space ${ }^{22,24}$ Since both geometries feature the same lead width $d$,
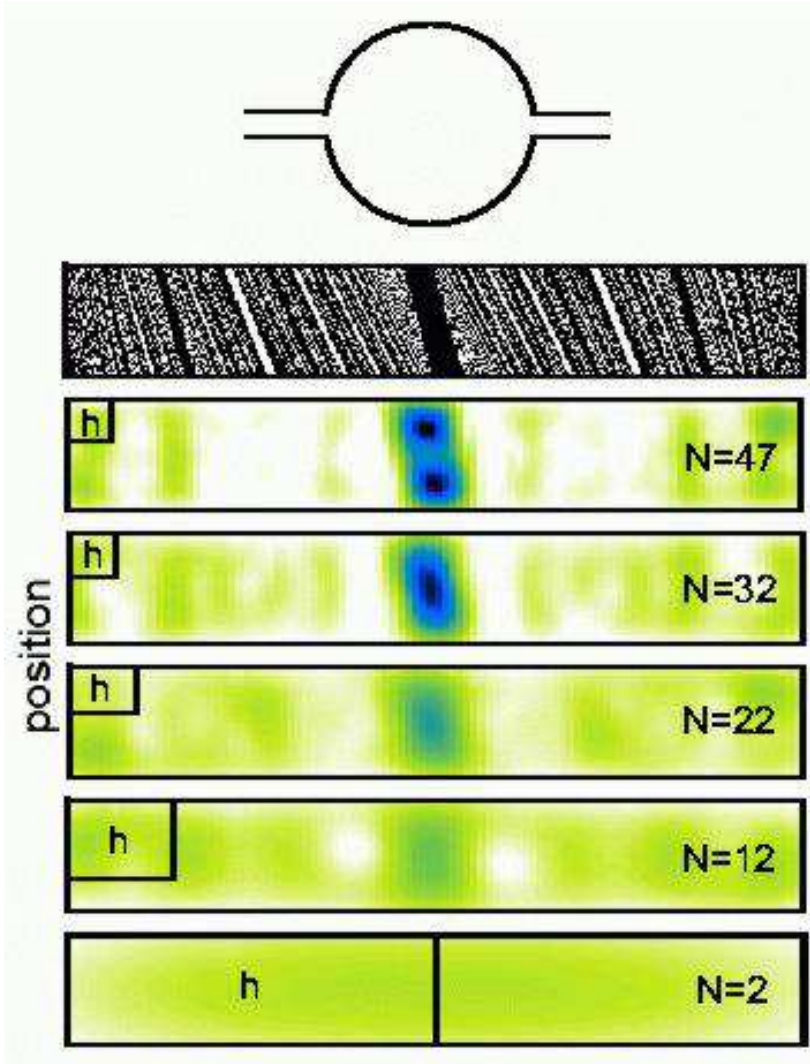

momentum

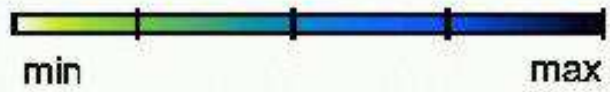

FIG. 3: (Color online) Circular billiard (no disorder). Top bar: Classical Poincaré surface of section (transmitted/reflected trajectories represented by black/white regions). Bottom bars: Cumulative Husimi distributions $H(x, p)$ of strongly transmitted scattering states (Eq. 5). $H(x, p)$ is shown for specific mode intervals $N$ and black frames indicate the size of the Planck cell $h$. For $N \gtrsim 24$ the largest transmission band (see central black region in the Poincaré surface) is larger than $h$ and can be resolved by the quantum scattering process. Above this threshold, noiseless scattering states appear in $H(x, p)$ in form of pronounced density enhancements near the largest transmission bands. 


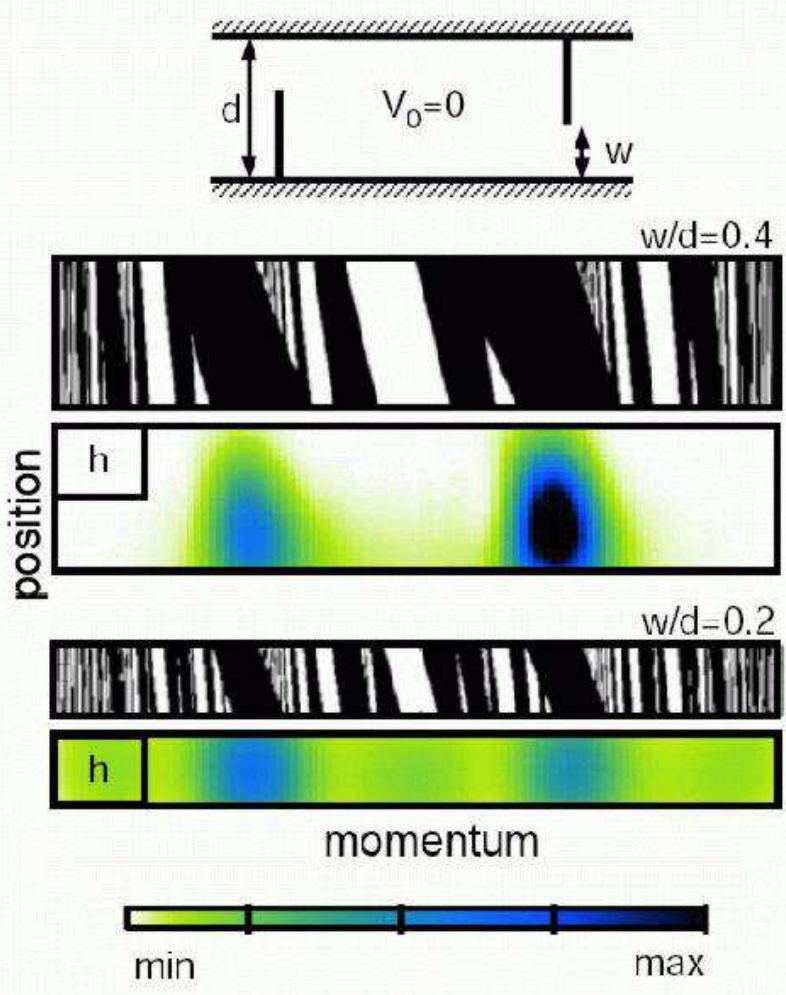

FIG. 4: (Color online) Rectangular billiard with tunable opening (no disorder, same color coding as in Fig. 3). Top two bars: Large shutter openings, $w / d=0.4$. Bottom two bars: Weakly open shutters, $w / d=0.2$. Decreasing the shutter opening below the threshold value $w / d \approx 0.32$ (where the size of the Planck cell $h$ is equal to the largest transmission band) reduces any pronounced enhancements in the Husimi distributions $H(x, p)$.

which controls the size of these transmission bands, noiseless states should appear at approximately the same $k_{F}$ for both cavities.

To further investigate this issue we demonstrate the quantum resolution of the classical phase space explicitly. To this end we compare Husimi distributions of scattering states with the Poincaré surface of section recorded at the entrance lead mouth. ${ }^{23,39}$ We calculate the cumulative Husimi function containing those eigenstates $\left|T_{i}\right\rangle$ of $t^{\dagger} t$ which correspond to the largest transmission eigenvalues $T_{i}$ within a given energy interval,

$$
H(x, p)=\sum_{i}^{M} H_{i}(x, p)=\sum_{i}^{M}\left|\left\langle T_{i} \mid x, p\right\rangle\right|^{2} .
$$

$|x, p\rangle$ is a coherent state of minimum uncertainty with its peak at the position $x, p$ and the number of eigenstates $\left|T_{i}\right\rangle$ that contribute to the above sum is chosen as $M=2 N$. In line with our calculations for the integrated eigenvalue distribution $I(T)$ (see Fig. 1), we now probe how electron energy, cavity opening, and disorder strength affect the distribution $H(x, p)$. In the circular

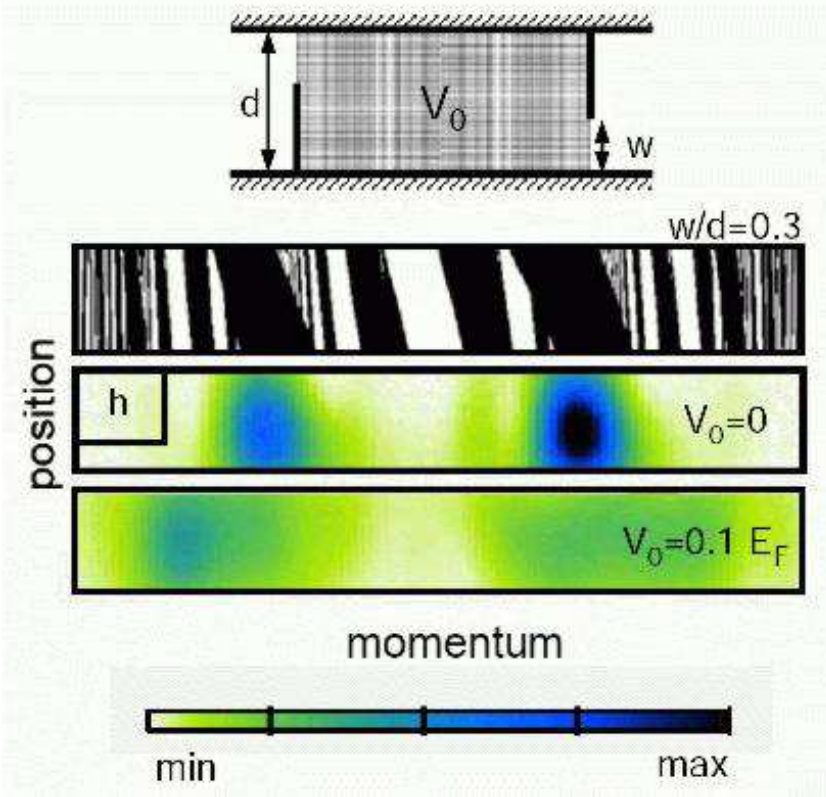

FIG. 5: (Color online) Rectangular billiard with tunable bulk disorder (fixed opening ratio $w / d=0.3$, same color coding as in Fig. 3). Top two bars: No disorder. Bottom bar: Moderate disorder, $V_{0}=0.1 E_{F}$. Bulk disorder destroys the appearance of noiseless states due to stochastic scattering.

billiard we probe the quantum-to-classical crossover by evaluating $H(x, p)$ in specific mode intervals $N$, corresponding to different electron energies (200 equidistant energy points per mode interval are calculated). We find that for low mode numbers $N$, the distribution $H(x, p)$ covers large parts of phase space more or less uniformly (see Fig. 3). For higher mode numbers, $H(x, p)$ shows a drastic enhancement near the largest transmission bands in phase space and a strongly reduced amplitude elsewhere. Comparing the size (area) of the Planck cell $h$ (indicated by the black frames in Fig. 3) to that of the largest transmission band (see the central black region in the classical Poincaré surface), we obtain an estimate for the threshold value above which noiseless scattering should appear. For the circular billiard this threshold is given by $k_{F} \approx 24 \pi / d$ (i.e. $N \approx 24$ ), at which value the largest transmission band and $h$ become equal in size $\stackrel{42}{=}$ This estimate indeed accurately predicts, above which value of $N$ our numerical results for $H(x, p)$ (Fig. 3) show significant enhancements near the largest transmission bands.

We now perform a similar analysis for the tunable rectangle. For this cavity we keep the electron energy fixed in the averaging interval $k_{F} \in[40.1,40.85] \times \pi / d$ and vary the cavity opening $w$ or, alternatively, the disorder strength $V_{0}$. By tuning the cavity openings at fixed energy we change the size of the largest transmission band at a fixed value of $h$. When these two phase space areas are equal in size, we obtain a threshold value for the appearance of the first noiseless state in terms of 
the cavity opening: $w / d \approx 0.32$. Comparing this estimate with our numerical results for $H(x, p)$ (see Fig. (4) yields again very good agreement: Whereas for an opening of $w / d=0.2<0.32$ the Husimi function $H(x, p)$ looks rather flat (Fig. 4 bottom), very clear enhancements around the largest transmission band appear for $w / d=0.4>0.32$ (Fig. 4 top). In Fig. 5 we demonstrate that bulk disorder in the cavity destroys any noiseless states by strongly reducing any pronounced enhancements which would otherwise be present in $H(x, p)$. We finally note that in all of the above cases (Figs. 3.5) drastic enhancements in $H(x, p)$ always come along with a corresponding offset in the integrated eigenvalue distribution $I(T)$ and vice versa. This evidence should unambiguously document the presence of noiseless scattering states in 2D-cavities.

To summarize, we have identified signatures of ballistic and stochastic scattering in the quantum-to-classical crossover of clean and disordered samples. A model for the transmission eigenvalue distribution $P(T)$ is proposed which combines previous approaches for the ballis- tic and disordered limit $\stackrel{24,26}{ }$ and which allows to extract contributions of different noise sources to our numerical results for $P(T)$. We provide the first evidence for "noiseless scattering states" 22 in clean, genuine $2 \mathrm{D}$-cavities and confirm the corresponding decomposition of the electronic flow in a classical and a quantum component ${ }^{24}$ The emergence of noiseless states is found to be determined by the size of the largest classical transmission band ${ }^{38}$ in phase space. The latter quantity, in turn, depends on the system specific geometry of the cavity and not necessarily on its chaoticity or on the lack thereof. In the presence of bulk disorder, noiseless scattering states disappear due to stochastic scattering, as previously anticipated $\underline{26}$

We thank E.V. Sukhorukov for useful suggestions and V. A. Gopar, H. Schomerus, A. D. Stone, B. Weingartner for helpful comments and discussions. Support by the FWF-Austria (Grants Nos. FWF-SFB016 and FWFP17359) and by the Max-Kade Foundation (New York) are gratefully acknowledged.
1 W. Schottky, Ann. Phys. (Leipzig) 57, 541 (1918).

2 C. W. J. Beenakker and Ch. Schönenberger, Physics Today 56(5), 37 (2003).

3 Ya. M. Blanter and M. Büttiker, Phys. Rep. 336, 1 (2000).

4 A. H. Steinbach, J. M. Martinis, and M. H. Devoret, Phys. Rev. Lett. 76, 3806 (1996)

5 R. J. Schoelkopf, P. J. Burke, A. A. Kozhevnikov, D. E. Prober, and M. J. Rooks, Phys. Rev. Lett. 78, 3370 (1997).

${ }^{6}$ M. Henny, S. Oberholzer, C. Strunk, and C. Schönenberger, Phys. Rev. B 59, 2871 (1999).

7 S. Oberholzer, E. V. Sukhorukov, C. Strunk, C. Schönenberger, T. Heinzel, and M. Holland, Phys. Rev. Lett. 86, 2114 (2001).

8 S. Oberholzer, E.V. Sukhorukov, and C. Schönenberger, Nature 415, 765 (2002).

9 B. Reulet, J. Senzier, and D. E. Prober, Phys. Rev. Lett. 91, 196601 (2003); Yu. Bomze, G. Gershon, D. Shovkun, L. S. Levitov, M. Reznikov, Phys. Rev. Lett. 95, 176601 (2005).

10 O. N. Dorokhov, Solid State Commun. 51, 381 (1984).

11 Y. Imry, Europhys. Lett. 1, 249 (1986).

12 V. A. Khlus, Sov. Phys. JETP 66, 1243 (1987); G. B. Lesovik, JETP Lett. 49, 592 (1989); M. Büttiker, Phys. Rev. Lett. 65, 2901 (1990).

13 K. E. Nagaev, Phys. Lett. A 169, 103 (1992); C. W. J. Beenakker and M. Büttiker, Phys. Rev. B 46, 1889 (1992).

14 K. E. Nagaev, Phys. Rev. B 52, 4740 (1995); V. I. Kozub and A. M. Rudin, Phys. Rev. B 527853 (1995); M. J. M. de Jong and C. W. J. Beenakker, in Mesoscopic Electron Transport, edited by L. P. Kouwenhoven, G. Schön, and L. L. Sohn, NATO ASI, Series E, Vol. 345 (Kluwer Academic, Dordrecht, 1996).

15 M. Leadbeater, V. I. Falko, and C. J. Lambert, Phys. Rev. Lett. 81, 1274 (1998).

16 O. Agam, I. Aleiner, and A. Larkin, Phys. Rev. Lett. 85, 3153 (2000).

17 Ya. M. Blanter and E. V. Sukhorukov, Phys. Rev. Lett. 84,
$1280(2000)$.

18 Ya. M. Blanter, H. Schomerus, and C. W. J. Beenakker, Physica E 11, 1 (2001).

19 H.-S. Sim and H. Schomerus, Phys. Rev. Lett. 89, 066801 (2002).

20 R. G. Nazmitdinov, H.-S. Sim, H. Schomerus, and I. Rotter, Phys. Rev. B 66, 241302(R) (2002).

21 M. Macucci, G. Iannaccone, G. Basso, and B. Pellegrini, Phys. Rev. B 67, 115339 (2003).

22 P. G. Silvestrov, M. C. Goorden, and C. W. J. Beenakker, Phys. Rev. B 67, 241301(R) (2003).

23 J. Tworzydło, A. Tajic, H. Schomerus, and C. W. J. Beenakker, Phys. Rev. B 68, 115313 (2003).

$24 \mathrm{Ph}$. Jacquod and E. V. Sukhorukov, Phys. Rev. Lett. 92, 116801 (2004).

25 F. Aigner, S. Rotter, and J. Burgdörfer, Phys. Rev. Lett. 94, 216801 (2005).

26 E. V. Sukhorukov and O. M. Bulashenko, Phys. Rev. Lett. 94, 116803 (2005).

27 O. M. Bulashenko, J. Stat. Mech. P08013 (2005).

${ }^{28} \mathrm{Ph}$. Jacquod and R. S. Whitney, Phys. Rev. B 73, 195115 (2006); Proceedings of the Fourth International Conference on Unsolved Problems of Noise and Fluctuations in Physics, Biology, and High Technology, AIP Conf. Proc. No. 800 AIP, Melville, NY, 2005, p. 225.

29 S. Rahav and P. W. Brouwer, Phys. Rev. B 73, 035324 (2006).

30 P. Marconcini, M. Macucci, G. Iannaccone, B. Pellegrini, G. Marola, Europhys. Lett. 73, 574 (2006).

31 F. W. J. Hekking and J. P. Pekola, Phys. Rev. Lett. 96, 056603 (2006).

32 V. A. Gopar, S. Rotter, and H. Schomerus, Phys. Rev. B 73, 165308 (2006).

33 J. Feist, A. Bäcker, R. Ketzmerick, S. Rotter, B. Huckestein, and J. Burgdörfer, Phys. Rev. Lett. 97, 116804 (2006).

34 S. Rotter, et al., Phys. Rev. B 62, 1950 (2000); 68, 165302 
(2003).

35 H. Lee, L. S. Levitov, and A. Yu. Yakovets, Phys. Rev. B 51, 4079 (1995).

${ }^{36}$ H. U. Baranger and P. A. Mello, Phys. Rev. Lett. 73, 142 (1994); R. A. Jalabert, J.-L. Pichard, and C. W. J. Beenakker, Europhys. Lett. 27, 255 (1994).

37 G. M. Zaslavsky, Phys. Rep. 80, 157 (1981).

38 L. Wirtz, J.-Z. Tang, and J. Burgdörfer, Phys. Rev. B 56, 7589 (1997); 59, 2956 (1999).

39 B. Weingartner, S. Rotter, J. Burgdörfer, Phys. Rev. B 72, 115342 (2005).

${ }^{40}$ We expect corrections to our phenomenological model, Eq. (4), to become important for intermediate to large dis- order strength, where a separation of phase space into two distinguishable components breaks down.

41 The cavity area $A=4+\pi$, and $d / \sqrt{A} \approx 0.09$ for both the circular and the stadium shaped cavity. With this choice of cavity sizes the stadium with a quadratic rectangular part features two half-circles on either side with radius $r=1$ (in arbitrary length units).

42 Note that in the stadium billiard this threshold value is somewhat larger than in the circle $\left(k_{F} \approx 32 \pi / d\right)$, due to the larger geometrical distance between lead mouths in this geometry. 